\title{
Turbulent Flame Speeds of G-equation Models in Unsteady Cellular Flows
}

\author{
Y-Y $\operatorname{Liu}^{1} *$ J. Xin², Y. Yu \\ ${ }^{1}$ Department of Mathematics, National Cheng Kung University, Tainan 70101, Taiwan \\ ${ }^{2}$ Department of Mathematics, University of California, Irvine, CA 92697, USA
}

\begin{abstract}
We perform a computationl study of front speeds of G-equation models in time dependent cellular flows. The G-equations arise in premixed turbulent combustion, and are Hamilton-Jacobi type level set partial differential equations (PDEs). The curvature-strain Gequations are also non-convex with degenerate diffusion. The computation is based on monotone finite difference discretization and weighted essentially nonoscillatory (WENO) methods. We found that the large time front speeds lock into the frequency of time periodic cellular flows in curvature-strain G-equations similar to what occurs in the basic inviscid G-equation. However, such frequency locking phenomenon disappears in viscous G-equation, and in the inviscid Gequation if time periodic oscillation of the cellular flow is replaced by time stochastic oscillation.
\end{abstract}

Keywords and phrases: G-equations, front speed computation, cellular flows, frequency locking

Mathematics Subject Classification: 70H20, 76F25, 76M20

\section{Introduction}

Front propagation in turbulent combustion is a nonlinear multiscale dynamical process $[15,16,18,19]$. In gasoline engine, the burning velocity determines the engine efficiency and the combustion cycle. Hence to measure and study the flame propagation speed is a fundamental issue in combustion experiments and theory. The comprehensive governing equations for flame propagation involve Navier-Stokes equations coupled with transport equations, which express laws of the fluid dynamics and the chemical reactions respectively. Simplified geometric models, such as the level set G-equations $[15,17]$, are often more efficient in improving our understanding of such complex phenomenon.

In level set approach [14], the so-called G-equation in turbulent combustion is:

$$
G_{t}+\mathbf{V}(\mathbf{x}, t) \cdot D G+s_{L}|D G|=0,
$$

which describes the motion of the flame front at a prescribed flow velocity $\mathbf{V}(\mathbf{x}, t)$ and a constant speed $s_{L}$ along the normal direction of the flame front called the "laminar flame speed". When the flame front is planar and the flow velocity is at rest, the front propagates at the speed $s_{L}$. If the flow is in motion, the front is wrinkled in time and eventually propagates at an asymptotic speed $s_{T}$ in each specified

${ }^{*}$ Corresponding author. E-mail: yuyul@ncku.edu.tw 
direction, which is called the "turbulent flame speed". Through the framework of homogenization theory, the existence of the turbulent flame speed of (1.1) has been rigorously established for periodic flows $[3,20]$ and random flows $[8,11]$.

As fluid turbulence is known to cause corrugations of flames, additional modeling terms are often incorporated into the basic G-equation (1.1) on physical grounds [15]. We shall study turbulent flame speeds characterized by such extended G-equation models involving curvature, diffusion or strain effects. The curvature G-equation is:

$$
G_{t}+\mathbf{V}(\mathbf{x}, t) \cdot D G+s_{L}|D G|=d|D G| \operatorname{div}\left(\frac{D G}{|D G|}\right)
$$

which comes from adding mean curvature term to the basic motion law. If the curvature terms is linearized, we arrive at the viscous G-equation:

$$
G_{t}+\mathbf{V}(\mathbf{x}, t) \cdot D G+s_{L}|D G|=d \Delta G
$$

which is also a model for understanding the numerical diffusion [14]. The strain G-equation is:

$$
G_{t}+\mathbf{V}(\mathbf{x}, t) \cdot D G+\left(s_{L}+d \frac{D G \cdot D \mathbf{V} \cdot D G}{\left|D G^{2}\right|}\right)|D G|=d|D G| \operatorname{div}\left(\frac{D G}{|D G|}\right) .
$$

The strain term $n \cdot D \mathbf{V} \cdot n$ comes from the flame surface stretching rate. We will give a brief derivation of all G-equation models later.

Our goal is to study turbulent flame speed $s_{T}$ and its dependence on the velocity field $\mathbf{V}(\mathbf{x}, t)$. Cellular flows are two dimensional vortical flows with nontrivial geometric streamline structures, hence often adopted for mathematical and computational study of $s_{T}$. Let us first consider the steady cellular flow:

$$
\mathbf{V}(\mathbf{x}, t)=A \cdot(-\sin (2 \pi x) \cos (2 \pi y), \cos (2 \pi x) \sin (2 \pi y)) .
$$

which is a two dimensional periodic incompressible flow with parameter $A$ as the flow intensity. The streamline of the cellular flow consist of a periodic array of hyperbolic (saddle points, separatrices) and elliptic (vortical) regions. For inviscid G-equation, $[1,13]$ showed that $s_{T}$ is enhanced by the cellular flow with growth rate $s_{T}=O(A / \log A)$. For viscous G-equation, it is proved in [9] that the diffusion term causes strong front speed bending (saturation), and the growth of $s_{T}$ slows down to uniform bound: $s_{T}=O(1)$. For curvature and strain G-equations, although the analytical justifications are still lacking, numerical results [10] showed that the curvature term causes weaker front speed bending than the regular diffusion term. Also the strain rate term causes flame quenching, that is, $s_{T}$ starts to decrease for larger $A$ and eventually drops to zero.

Next, we consider the unsteady (time dependent) cellular flow. For inviscid G-equation (1.1) and time periodic cellular flow, it is observed in [2] that the front propagation may be synchronized by the spatial and temporal periodicity, and $s_{T}$ is approximately frequency times a rational number. Such phenomenon is called "frequency locking". The time periodic and random cellular flows are also considered in [12] within a reaction-diffusion-advection (RDA) equation model where temporally stochastic perturbations of the cellular flow also cause strong resonance (although the number of resonance peaks reduces to one).

In this paper, we study turbulent flame speeds in unsteady cellular flows for extended G-equation models (1.2),(1.3),(1.4). The organization is as follows. In section 2, we give an overview of G-equation models and refer the associated numerical schemes based on monotone finite difference methods to [10]. In section 3, we show numerical findings. For time periodically shifted cellular flows, we found that frequency locking of the front propagation persists in curvature and strain G-equations, and the locking phenomenon is robust with respect to the coefficient $d$ of the curvature or strain term. On the other hand, it disappears in the viscous G-equation (1.3), and in time random cellular flows where $s_{T}$ decreases in the oscillation "frequency" (the reciprocal of correlation length). For time periodically amplified cellular flows, all results are similar except that if the amplitude oscillation frequency is too high then the front becomes insensitive to the cellular flow. We end with concluding remarks in section 4, and acknowledgements in section 5 . 


\section{G-equation Models}

In the thin reaction zone regime and the corrugated flamelet regime of premixed turbulent combustion [15], the flame front is modeled by a level set function $\{G(\mathbf{x}, t)=0\}$, which is the interface between the unburned fuel $\{G<0\}$ and burned fuel $\{G>0\}$. The trajectory of a particle $\mathbf{x}(t)$ on the interface is given by a velocity field and a laminar speed:

$$
\frac{\mathrm{d} \mathbf{x}}{\mathrm{d} t}=\mathbf{V}(\mathbf{x}, t)+s_{L} n
$$

where the positive constant $s_{L}$ is called the laminar flame speed and $n=D G /|D G|$ is the normal velocity ( $D$ : spatial gradient). In terms of $G$, the motion law (2.1) gives the inviscid G-equation (1.1). To take account of the effect of flame stretching, the surface stretch rate may be added as a first order correction term on laminar flame speed:

$$
\begin{gathered}
\hat{s}_{L}=s_{L}-d(\kappa+\mathcal{S}), \\
\kappa=\operatorname{div}(n), \mathcal{S}=-n \cdot D \mathbf{V} \cdot n .
\end{gathered}
$$

Here $d$ is called the Markstein diffusive number, $\kappa$ is the mean curvature and $\mathcal{S}$ is called the strain rate. Replacing $s_{L}$ by $\hat{s}_{L}$ in the motion law (2.1), we obtain the strain G-equation (1.4). If we include the motion by curvature only, we have the curvature G-equation (1.2). If the curvature term is further linearized, we arrive at the viscous G-equation (1.3).

Suppose the flame front propagates in $x$-direction in two dimensional space $(\mathbf{x}=(x, y))$. Consider the stripe domain $\mathbb{R} \times[0,1]$, and the burned region at time $t$ is $\{\mathbf{x} \in \mathbb{R} \times[0,1]: G(\mathbf{x}, t)<0\}$. Denote $\mathcal{A}(t)$ the area that the burned region has invaded during time interval $(0, t)$ :

$$
\mathcal{A}(t)=\int_{\mathbb{R} \times[0,1]} \chi_{\{G(\mathbf{x}, t)<0\}}-\chi_{\{G(\mathbf{x}, 0)<0\}} \mathrm{d} \mathbf{x} .
$$

( $\chi$ : indicator function.) We refer $\mathcal{A}^{\prime}(t)$ as the instantaneous burning velocity. Then turbulent flame speed is the large time linear growth rate of $\mathcal{A}(t)$ or the average of $\mathcal{A}^{\prime}(t)$ :

$$
s_{T}=\lim _{t \rightarrow \infty} \frac{\mathcal{A}(t)}{t}=\lim _{t \rightarrow \infty} \frac{1}{t} \int_{0}^{t} \mathcal{A}^{\prime}(t) d t .
$$

In numerical simulation, consider G-equation with initial data $G(\mathbf{x}, 0)=x$. If $\mathbf{V}(\mathbf{x}, t)$ is spatially periodic, we can reduce the computational domain to $[0,1]^{2}$ by imposing the affine periodic condition:

$$
\left\{\begin{array}{ll}
G_{t}+\mathbf{V}(\mathbf{x}, t) \cdot D G+s_{L}|D G|=0 & \text { in } \mathbb{T}^{2} \times(0, \infty) \\
G(\mathbf{x}, 0)=x & \text { on } \mathbb{T}^{2} \times\{t=0\} \\
G\left(\mathbf{x}+k \mathbf{e}_{1}, t\right)=G(\mathbf{x}, t)+k & \mathbf{x} \in[0,1]^{2}, k \in \mathbb{Z}
\end{array} .\right.
$$

Based on the framework of finite difference computation of Hamilton-Jacobi equations [14], the first order derivatives (flow velocity, laminar speed and strain terms) are discretized as monotone numerical Hamiltonian and approximated by weighted essentially nonoscillatory (WENO) scheme. The second order derivatives (curvature and diffusion terms) are approximated by central differencing. For the explicit time step discretization, total variation diminishing Runge-Kutta (TVD RK) scheme is used, and the time step size restriction is given by the CFL condition. See [10] for details of the numerical schemes. We compute (2.3) with spatial grid size $200 \times 200$.

\section{Numerical Results}

Our first set of numerical results is on the time periodically shifted cellular flow:

$$
\mathbf{V}(\mathbf{x}, t)=A \cdot(-\sin (2 \pi x+B \sin (2 \pi \omega t)) \cos (2 \pi y), \cos (2 \pi x+B \sin (2 \pi \omega t)) \sin (2 \pi y)) .
$$



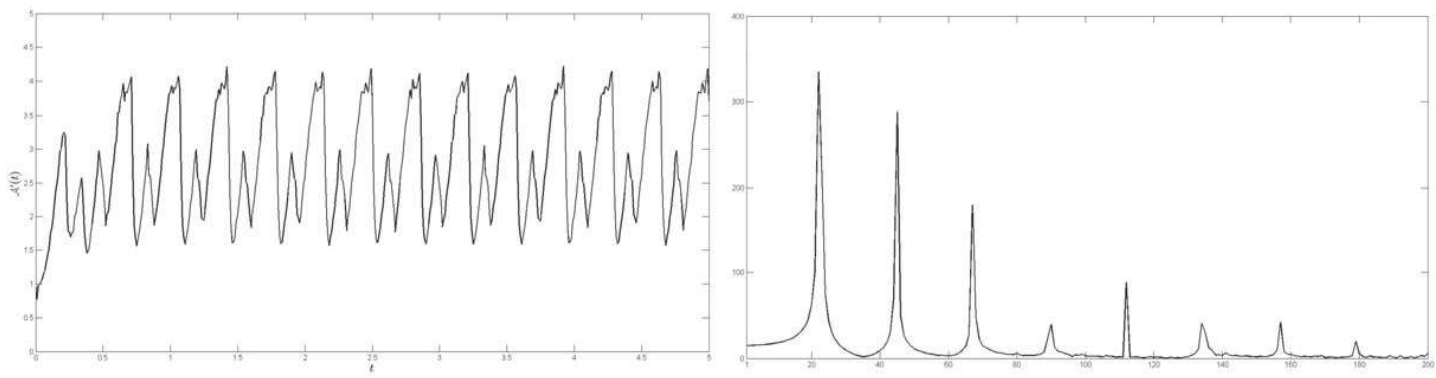

Figure 1. Left: Plot of $\mathcal{A}^{\prime}(t)$ for inviscid G-equation with time periodically shifted cellular flow, $s_{L}=1, A=4, B=1, \omega=2.8$. Right: plot of the absolute values of the first 200 components of the 1024 point discrete Fourier transform of $\mathcal{A}^{\prime}(t), t \in(2,10)$. An array of equally spaced peak pattern appears.
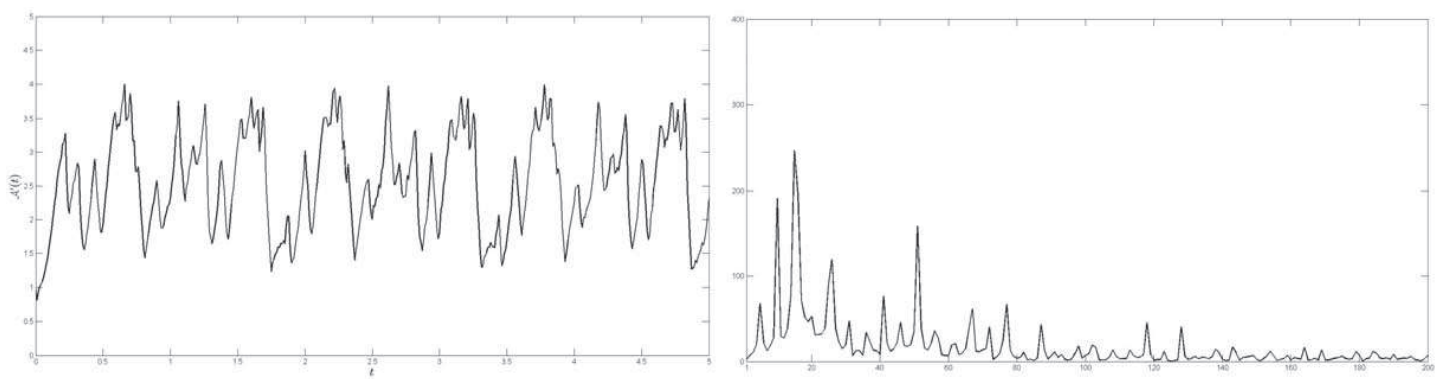

Figure 2. Left: Plot of $\mathcal{A}^{\prime}(t)$ with $\omega=3.2$. Right: Fourier spectral plot. The spectral peaks are less regularly distributed as in the right panel of Fig. 1.

The flow oscillates temporally in the $x$-direction with amplitude $B$ and frequency $\omega$. We fix $s_{L}=1$, $A=4, B=1$ and choose different values for $d$ to see the effect of the curvature, diffusion and strain terms. Note that if $d=0$ then all G-equations are identical to inviscid G-equation, and we choose $d$ small enough to avoid the strain term overpowering $s_{L}$.

In figure 1 , the left panel is the plot of $\mathcal{A}^{\prime}(t)$ for inviscid G-equation with $\omega=2.8$. Here $\mathcal{A}^{\prime}(t)$ evolves into a periodic function after a transient time. To confirm this, we take the discrete Fourier transform of $\mathcal{A}^{\prime}(t)$ at 1024 uniform sample points over the time interval $(2,10)$, and plot the absolute values of the first 200 Fourier components in the right panel. An array of equally spaced sharp peaks appear and lend support for time periodicity. In this case frequency locking occurs, and we can simply compute $s_{T}$ by taking the average of $\mathcal{A}^{\prime}(t)$ over a few periods. The left panel of figure 2 is a plot of $\mathcal{A}^{\prime}(t)$ at $\omega=3.2$, the absolute values of the first 200 Fourier components are shown in the right panel where we notice a mixture of unequally spaced large peaks and many irregular smaller peaks. The distinct equally spaced peak pattern is absent, suggesting non-periodicity. The $s_{T}$ is evaluated by taking average of $\mathcal{A}^{\prime}(t)$ over a large time interval as $(2.2)$.

The frequency locking effect can be understood as follows. In the transient time, the front propagation structure is synchronized with the temporal and spatial periodicity of the cellular flow and evolves into a periodic steady state. During a multiple of the oscillatory period $\Delta t=M \cdot 1 / \omega$, the flame front translates through a multiple of the cell $\Delta \mathcal{A}(t)=N$. ( $N, M$ : integers.) Then the turbulent flame speed is:

$$
s_{T}=\frac{\Delta \mathcal{A}(t)}{\Delta t}=\frac{N}{M} \cdot \omega .
$$


Figure 3 shows the plot of turbulent flame speed as a function of the oscillatory frequency $s_{T}=s_{T}(\omega)$ for all G-equation models with various $d$. For inviscid, curvature and strain G-equations (1.1),(1.2),(1.4), frequency locking is observed. Here $s_{T}(\omega)$ is piecewise linear with rational slopes $r=s_{T} / \omega$. In each linear segment the front propagation locks into the same periodicity pattern, that is, the same $N, M$ in (3.1) and hence same $r$. Between the linear segments, the locking effect loses stability and $s_{T}(\omega)$ decreases. Notice that frequency locking phenomenon is also robust with respect to $d$, that is, $s_{T}$ may be the same even as $d$ varies (different curves may coalesce on bold line segments of top and middle panels of Figure $3)$. This is different from that in steady cellular flow where $s_{T}$ is strictly decreasing as $d$ increases [10].

For viscous G-equation, however, no frequency locking appears. Instead the dissipation effect causes the level set function to converge to the stationary solution:

$$
G(\mathbf{x}, t)=-s_{T} t+x+u(\mathbf{x}, t)
$$

with $u(\mathbf{x}, t)$ a periodic function in $[0,1]^{2} \times[0,1 / \omega]$. In the plot of $s_{T}(\omega)$, we see two resonant peaks for smaller $d$, then the peaks start to merge and disappear for larger $d$.

Our second set of numerical results is when $\mathbf{V}$ is the time periodically amplified cellular flow:

$$
\mathbf{V}(\mathbf{x}, t)=A \sin (2 \pi \omega t) \cdot(-\sin (2 \pi x) \cos (2 \pi y), \cos (2 \pi x) \sin (2 \pi y)) .
$$

Figure 4 is the plot $s_{T}=s_{T}(\omega)$ for all G-equation models and various $d$. When $\omega$ is small, all results are similar to the time periodically shifted cellular flow. When $\omega$ is larger, however, the direction of the flow changes so fast in time that the flame front cannot be really wrinkled, and so remains almost planar. Hence $s_{T}(\omega)$ is not much responsive to the cellular flow patterns, and decreases to $s_{L}$ as $\omega \rightarrow+\infty$ in all the G-equations. Similar phenomenon of front speed reduction due to fast temporal oscillation of the flow is known in reaction-diffusion-advection models [4-7].

Finally we consider the time stochastically shifted cellular flow:

$$
\mathbf{V}(\mathbf{x}, t)=A \cdot(-\sin (2 \pi x+\gamma(t)) \cos (2 \pi y), \cos (2 \pi x+\gamma(t)) \sin (2 \pi y)) .
$$

Here $\gamma(t)$ is the Ornstein-Uhlenbeck process defined by the Ito equation:

$$
\mathrm{d} \gamma(t)=-\alpha \gamma(t) \mathrm{d} t+\beta \mathrm{d} W(t),
$$

where $W(t)$ is the Wiener process, $\alpha$ and $\beta$ are positive constants. We choose $\beta=\sqrt{2} \alpha^{3 / 4}$ so that the power spectrum is invariant in $\alpha$, the reciprocal of the correlation length of $\gamma(t)$. Figure 5 shows the plot $s_{T}(\alpha)$ for inviscid G-equation. We see that $s_{T}$ always decreases as $\alpha$ increases, stochasticity in time kills the resonance phenomenon. This is different to that in RDA equation model where one wider resonance peak still remains under time stochastic perturbation of cellular flows [12].

We remark that the intensity (in the space-time root mean square sense) of all unsteady cellular flows studied here is held fixed as we vary temporal oscillation frequency or parameter $\alpha$ in the random case. If the temporal oscillation frequency or $\alpha$ is fixed while the flow intensity is increased, then $s_{T}$ is expected to increase.

\section{Concluding Remarks}

We have studied numerically the basic and the curvature-strain dependent G-equation models and their corresponding turbulent flame speeds in various unsteady cellular flows. Numerical results indicate that the frequency locking phenomenon occurs in inviscid, curvature and strain G-equations but not in viscous G-equations. It also disappears in time stochastically shifted cellular flows.

In future work, we plan to study more general unsteady (time periodic or random) flows, including three dimensional flows for G-equation models.

Acknowledgements. The work was partially supported by NSC grants (YL) of Taiwan, NSF grants DMS-0911277, DMS-1211179 (JX); DMS-0901460, CAREER award DMS-1151919 (YY) of USA. 

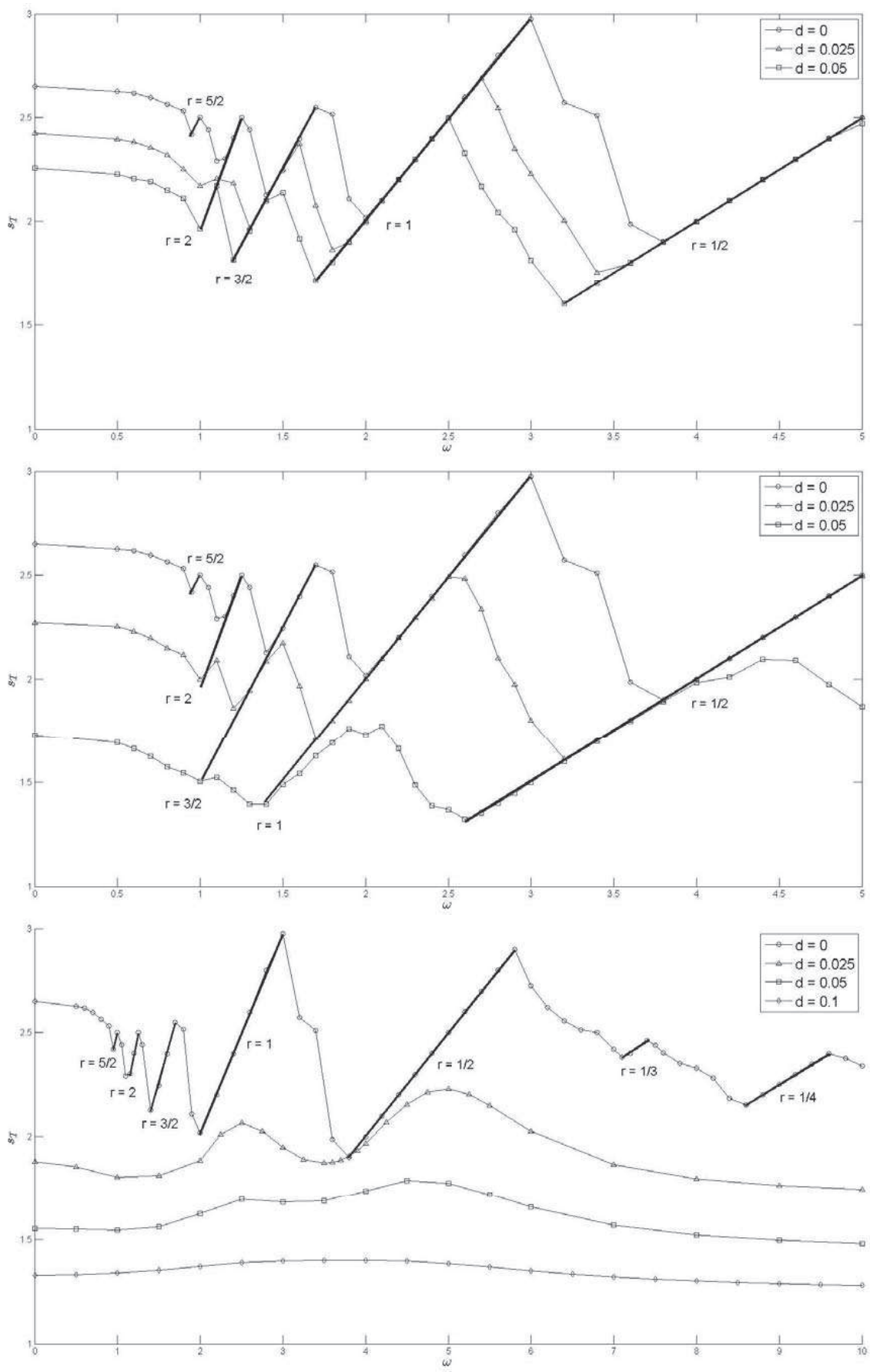

Figure 3. Turbulent flame speeds of G-equation models with time periodically shifted cellular flow. Above: inviscid/curvature G-equations. Middle: inviscid/strain Gequations. Below: inviscid/viscous G-equations. The bold lines indicate the frequency locking pattern $r=s_{T} / \omega$. 

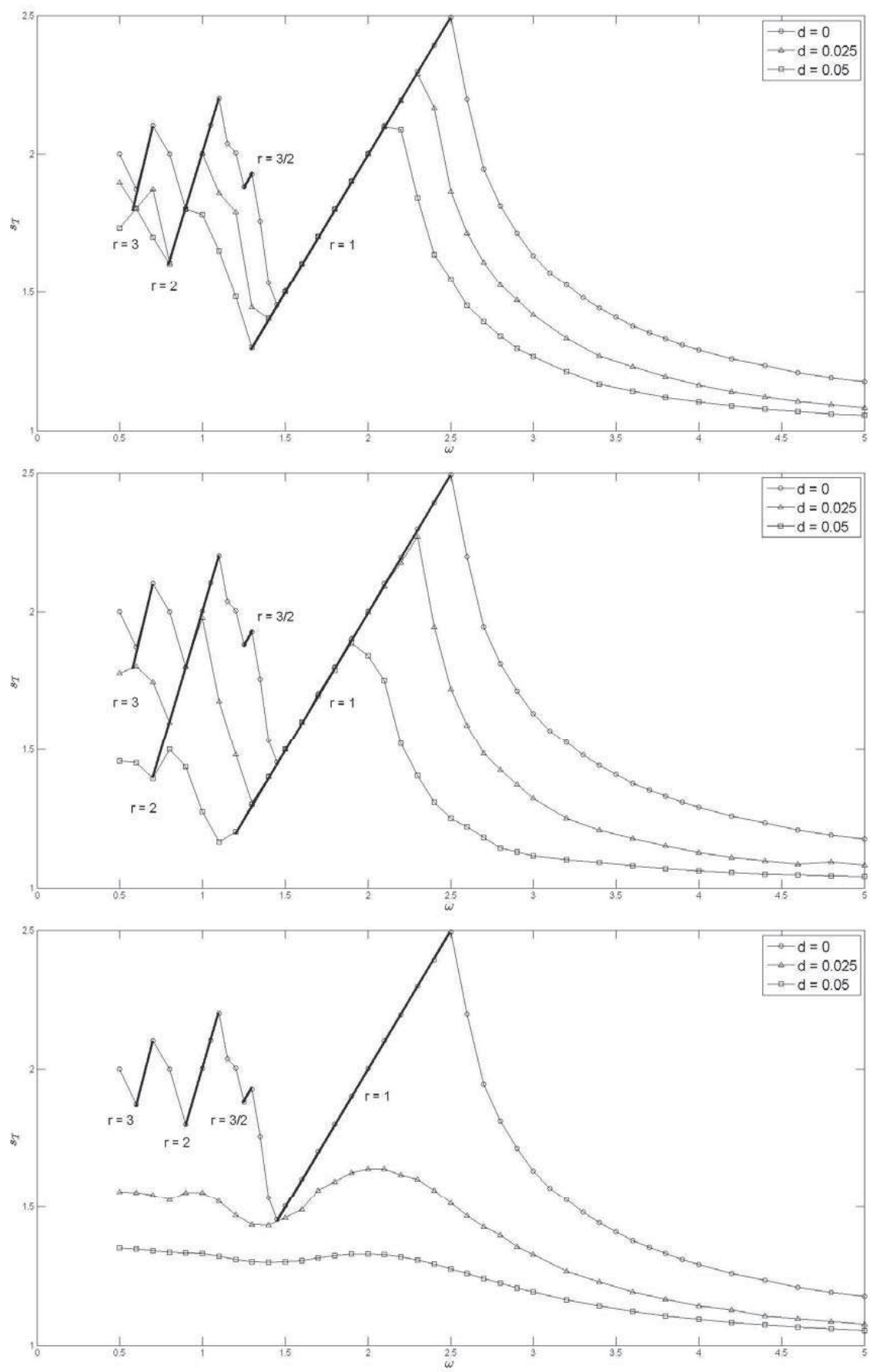

Figure 4. Turbulent flame speeds of G-equation models with time periodically amplified cellular flow. Above: inviscid/curvature G-equations. Middle: inviscid/strain Gequations. Below: inviscid/viscous G-equations. The bold lines indicate the frequency locking pattern $r=s_{T} / \omega$. 


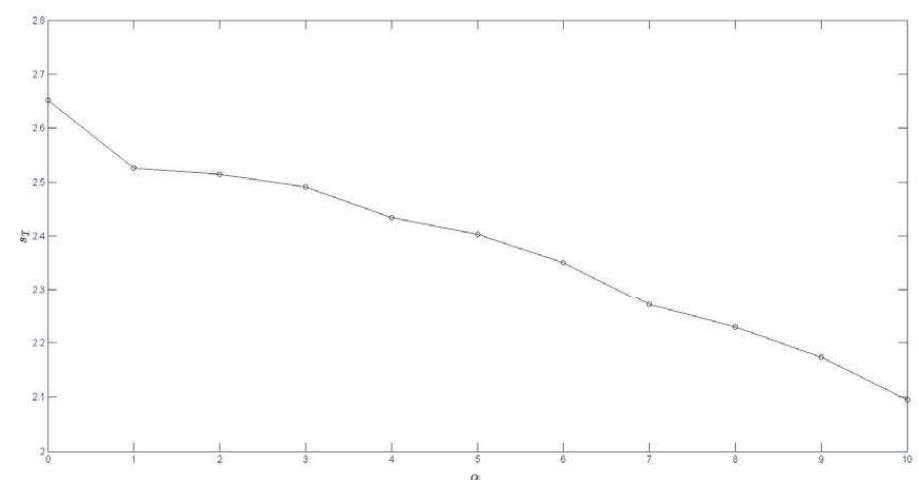

Figure 5. Turbulent flame speeds of inviscid G-equation with Ornstein-Uhlenbeck process being time oscillatory shift in cellular flow, as a function of $\alpha$, the reciprocal of the correlation length, or "oscillation frequency".

\section{References}

[1] M. Abel, M. Cencini, D. Vergni, A. Vulpiani. Front Speed Enhancement in Cellular Flows. Chaos 12 (2002), $481-488$.

[2] M. Cencini, A. Torcini, D. Vergni, A. Vulpiani. Thin front propagation in steady and unsteady cellular flows. Phys. Fluids 15 (2003), 679-688.

[3] P. Cardaliaguet, J. Nolen, P. E. Souganidis. Homogenization and Enhancement for the G-Equation. Arch. Rational Mech. Analysis 199 (2011), 527-561.

[4] B. Denet. Possible Role of Temporal Correlations in the Bending of Turbulent Flame Velocity. Combust. Theory Model. 3 (1999), 585-589.

[5] B. Khouider, A. Bourlioux, A. Majda. Parametrizing Turbulent Flame Speed-Part I: Unsteady Shears, Flame Residence Time and bending. Combust. Theory Model. 5 (2001), 295-318.

[6] J. Nolen, J. Xin. Reaction-Diffusion Front Speeds in Spatially-Temporally Periodic Shear Flows. SIAM J. Multiscale Modeling and Simulation 1 (2003), 554-570.

[7] J. Nolen, J. Xin. Asymptotic Spreading of KPP Reactive Fronts in Incompressible Space-Time Random Flows. Ann Inst. H. Poincaré, Analyse Non Lineaire 26 (2009), 815-839.

[8] P. Cardaliaguet, P. E. Souganidis. Homogenization and Enhancement of the G-equation in Random Environments. Comm. Pure Appl. Math, to appear.

[9] Y.-Y. Liu, J. Xin, Y. Yu. Asymptotics for turbulent flame speeds of the viscous G-equation enhanced by cellular and shear flows. Arch. Rational Mech. Analysis 202 (2011), 461-492.

[10] Y.-Y. Liu, J. Xin, Y. Yu. A Numerical Study of Turbulent Flame Speeds of Curvature and Strain G-equations in Cellular Flows. Physica D 243 (2013), 20-31.

[11] J. Nolen, A. Novikov. Homogenization of the G-equation with incompressible random drift in two dimensions. Comm. Math Sci. 9 (2011), 561-582.

[12] J. Nolen, J. Xin. Computing reactive front speeds in random flows by variational principle. Physica D 237 (2008), $3172-3177$.

[13] A. Oberman. Ph.D. thesis, University of Chicago, Chicago, IL, 2001.

[14] S. Osher, R. Fedkiw. Level Set Methods and Dynamic Implicit Surfaces. Springer-Verlag, New York, NY 2002.

[15] N. Peters. Turbulent Combustion. Cambridge University Press, 2000.

[16] P.D. Ronny. Some Open Issues in Premixed Turbulent Combustion. Lecture Notes in Physics 449 (1995), 3-22.

[17] F. Williams, Turbulent Combustion. The Mathematics of Combustion (J. Buckmaster, ed.), SIAM, Philadelphia (1985) 97-131.

[18] J. Xin, Front Propagation in Heterogeneous Media. SIAM Review 42 (2000), 161-230.

[19] J. Xin. An Introduction to Fronts in Random Media. Surveys and Tutorials in the Applied Mathematical Sciences 5, Springer, 2009.

[20] J. Xin, Y. Yu. Periodic Homogenization of Inviscid G-equation for Incompressible Flows. Comm. Math Sci. 8 (2010), $1067-1078$ 